\title{
INTEGRAL SLIDING MODE CONTROLLER OF A ROTATIONAL SERVODRIVE
}

\author{
M. Bouri, D. Thomasset, S. Scavarda \\ Laboratoire d'Automatique Industrielle \\ Institut National des Sciences Appliquées de Lyon \\ Bat 303, 20 Av. A.Einstein 69621 VILLEURBANNE \\ phone: (33) 72.43.81.98, fax: (33) 72.43.85.35 \\ e-mail: bouri@laifluide2.insa-lyon.fr
}

\begin{abstract}
In tracking or position control of an electropneumatic servodrive modelling errors like the leakage mass flow rates and the gravity torque or also the dry friction, constitute a serious constraint when designing a control law. We describe in this paper a robust control approach based upon a local change of coordinates and a nonlinear sliding mode control synthesis. An integral surface is used and this allows us to overcome the use of an accelerometer. Experimental tracking results are then related.
\end{abstract}

Keywords: Electropneumatic system. Non linear control. Sliding mode control. Integral surface.

\section{Principal Notations}

$\theta, \omega, \gamma \quad$ position, speed, acceleration,

$\mathrm{P}_{\mathrm{x}} \quad$ pressure in the chamber $\mathrm{x}$,

$\mathrm{P}_{\mathrm{s}}, \mathrm{P}_{\mathrm{c}} \quad$ supply and exhaust pressures,

$\mathrm{V}_{\mathrm{x}} \quad$ volume of the chamber $\mathrm{x}$,

$\mathrm{k} \quad$ polytropic constant,

r perfect gas constant,

$\mathrm{T}$ ambient temperature,

i interface current,

$\mathrm{K}_{\mathrm{c}} \quad$ torque constant,

$\mathrm{K}_{\theta} \quad$ volume constant,

$\mathrm{T}_{\text {dry }} \quad$ dry friction,

$\mathrm{M}_{\mathrm{b}}, \mathrm{J} \quad$ load and total inertia,

$\mathrm{W}, \lambda \quad$ switching gain and surface parameter,

$\phi \quad$ boundary layer thickness,

sup(.) upper bound function,

$\dot{m}_{p}\left(i, P_{p}\right)$ and $\dot{m}_{n}\left(i, P_{n}\right)$ are the mass flow rates provided by the servovalve flow stage to the cylinder chambers.

$\dot{m}_{v}$ is the interchamber leakage mass flow rates

$\mathrm{D}\left(\mathrm{P}_{\mathrm{s}}, \mathrm{P}_{\mathrm{x}}\right)$ and $\mathrm{D}\left(\mathrm{P}_{\mathrm{x}}, \mathrm{P}_{\mathrm{c}}\right)$ are the mass flow rate functions obtained from experimental results.

\section{Introduction}

The sliding mode or variable structure controllers are now well established and several applications in different fields are pointed out [2] [5] [7] [8] [9] [13]. The two major interests of this kind of control are the robustness with respect to the unmodelled dynamics and the possibility of imposing, on the sliding surface, a dynamic independent from the system behaviour.
This paper is organized as follows:

In Section 1 the servodrive, the non-linear model and the non linear affine state model are presented.

The control law is designed in section 2 in three steps. In the first step, using a local diffeomorphism, the nonlinear model is rewritten into a canonical form. This model does not contain the acceleration as a state variable. This is recovered thanks to the information of the pressure difference. In the second step, two sliding mode controllers are synthesized with and without an integral term in the switching surface. Finally, the analysis of the bounded modelling errors is carried out to fix the sliding mode control parameters.

Then, section 3 presents and discuss the tracking results obtained with and without the integral term. A compromise between the width of the boundary layer and the chattering in both the control and the output is carried out.

\section{The electropneumatic servodrive system}

The system under consideration is a rotational electropneumatic servodrive controlled by a five way servovalve (figure 1). The model of the above system is mainly obtained using three fundamental laws: chamber pressure evolution law, flow rate laws [1] [4] and mechanical equation.

$$
\begin{aligned}
& \frac{d P_{p}}{d t}=\frac{k r T}{V_{p}(\theta)}\left[\dot{m}_{p}\left(i, P_{p}\right)-\dot{m}_{v}\left(P_{p}, P_{n}\right)-\frac{K_{\theta}}{r T} P_{p} \omega\right] \\
& \frac{d P_{n}}{d t}=\frac{k r T}{V_{n}(\theta)}\left[\dot{m}_{n}\left(i, P_{n}\right)+\dot{m}_{v}\left(P_{p}, P_{n}\right)+\frac{K_{\theta}}{r T} P_{n} \omega\right] \\
& \frac{d \omega}{d t}=\frac{1}{J}\left[K_{c}\left(P_{p}-P_{n}\right)+M_{b} g l \sin (\theta)-T_{d r y}\right] \\
& \frac{d \theta}{d t}=\omega \\
& \text { where: * } V_{p}(\theta)=V_{p o}+K_{\theta} \theta * V_{n}(\theta)=V_{n o}-K_{\theta} \theta
\end{aligned}
$$




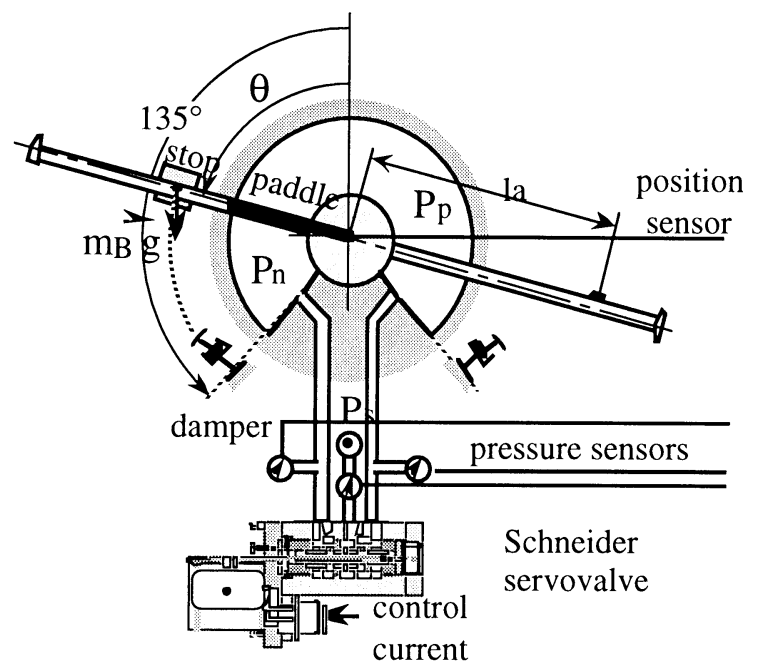

figure 1 - The electropneumatic system

In order to use the theoretical results about nonlinear analytic systems and by assuming the bridge symmetry Richard [10] has introduced a new control variable $A_{u}^{*}(i)$ given as follows:

$\begin{cases}\dot{\mathrm{m}}_{\mathrm{p}}\left(\mathrm{i}, \mathrm{P}_{\mathrm{p}}\right)=-\dot{\mathrm{m}}_{\mathrm{fp}}\left(\mathrm{P}_{\mathrm{p}}\right)+\varphi_{\mathrm{p}}\left(\mathrm{P}_{\mathrm{p}}, \operatorname{sign}\left(\mathrm{A}^{*}\right)\right) \mathrm{A}_{\mathrm{u}}^{*}(\mathrm{i}) \\ \dot{\mathrm{m}}_{\mathrm{n}}\left(\mathrm{i}, \mathrm{P}_{\mathrm{n}}\right)=-\dot{\mathrm{m}}_{\mathrm{fn}}\left(\mathrm{P}_{\mathrm{n}}\right)-\varphi_{\mathrm{n}}\left(\mathrm{P}_{\mathrm{n}}, \operatorname{sign}\left(\mathrm{A}^{*}\right)\right) \mathrm{A}_{\mathrm{u}}^{*}(\mathrm{i})\end{cases}$
where $\mathrm{A}^{*}(\mathrm{i})=\left\{\begin{array}{cc}\mathrm{A}_{\mathrm{sp}}(\mathrm{i})-\mathrm{A}_{\mathrm{fp}} & \text { if } \mathrm{i} \geq 0 \\ -\left(\mathrm{A}_{\mathrm{pe}}(\mathrm{i})-\mathrm{A}_{\mathrm{fp}}\right) & \text { if } \mathrm{i}<0\end{array}\right.$

and $\quad \mathrm{Au}_{\mathrm{u}}^{*}(\mathrm{i})=\mathrm{A}^{*}(\mathrm{i}) \mathrm{D}\left(\mathrm{P}_{\mathrm{s}}, \mathrm{Pe}\right)$

The functions $\varphi_{\mathrm{p}}\left(\mathrm{P}_{\mathrm{p}}, \operatorname{sign}\left(\mathrm{A}^{*}\right)\right)$ and $\varphi_{\mathrm{n}}\left(\mathrm{P}_{\mathrm{n}}, \operatorname{sign}\left(\mathrm{A}^{*}\right)\right)$ are given by the following equations:

$\varphi_{p}\left(P_{p}, \operatorname{sign}\left(A^{*}\right)\right)=\left\{\begin{array}{l}\frac{D\left(P_{s}, P_{p}\right)}{D\left(P_{s}, P_{e}\right)} \text { if } A^{*} \geq 0 \\ \frac{D\left(P_{p}, P_{e}\right)}{D\left(P_{s}, P_{e}\right)} \text { if } A^{*}<0\end{array}\right.$

$\varphi_{n}\left(P_{n}, \operatorname{sign}\left(A^{*}\right)\right)=\left\{\begin{array}{l}\frac{D\left(P_{s}, P_{n}\right)}{D\left(P_{s}, P_{e}\right)} \text { if } A^{*} \leq 0 \\ \frac{D\left(P_{n}, P_{e}\right)}{D\left(P_{s}, P_{e}\right)} \text { if } A^{*}>0\end{array}\right.$

More informations about this nonlinear model can be found in [10] and [11].

Combining the relations (1) to (6) we obtain the required nonlinear affine model :

$$
\left\{\begin{array}{l}
\dot{x}=f(x)+g(x) A_{u}^{*} \\
y=h(x)=\theta
\end{array}\right.
$$

$$
\begin{aligned}
& f(x)=\left(\begin{array}{c}
\frac{k r T}{V_{p}(\theta)}\left[-\dot{m}_{f p}\left(P_{p}\right)-\dot{m}_{v}\left(P_{p}, P_{n}\right)-\frac{K_{\theta}}{r T} P_{p} \omega\right] \\
\frac{k r T}{V_{n}(\theta)}\left[-\dot{m}_{f n}\left(P_{n}\right)+\dot{m}_{v}\left(P_{p}, P_{n}\right)+\frac{K_{\theta}}{r T} P_{n} \omega\right] \\
\frac{l}{J}\left[K_{c}\left(P_{p}-P_{n}\right)+M_{b} g l \sin (\theta)-T_{d r y}\right] \\
\omega
\end{array}\right) \\
& g(x)=\left(\begin{array}{c}
\frac{k r T}{V_{p}(\theta)} \varphi_{p}\left(P_{p}, \operatorname{sign}\left(A_{u}^{*}\right)\right) \\
-\frac{k r T}{V_{n}(\theta)} \varphi_{n}\left(P_{n}, \operatorname{sign}\left(A_{u}^{*}\right)\right) \\
0 \\
0
\end{array}\right)
\end{aligned}
$$

\section{Control synthesis}

\subsection{Coordinate transformation}

Let us consider the previous nonlinear affine model (7). The output $h(x)=\theta$ of this model has a relative degree $r=3$. Then, the new coordinates may be chosen as below:

$$
\left\{\begin{array}{l}
z_{1}=\theta \\
z_{2}=\omega \\
z_{3}=\frac{K_{c}}{J}\left(P_{p}-P_{n}\right) \\
z_{4}=P_{p}
\end{array}\right.
$$

It contains only the accessible variables and we easily check that this coordinate transformation is conform thanks to the property of the non-singularity of its jacobian matrix. In these new coordinates $z=\left(z_{1}, z_{2}, z_{3}, z_{4}\right)^{T}$, the system dynamic may be rewritten in the form:

$$
\left\{\begin{array}{l}
\dot{z}_{1}=z_{2} \\
\dot{z}_{2}=z_{3}+\delta \\
\dot{z}_{3}=\left(\alpha_{n}(z)+\Delta_{f}\right)+\beta_{n}(z) A_{u}^{*} \\
\dot{z}_{4}=\dot{P}_{p}
\end{array}\right.
$$

where $\alpha_{n}(z)$ and $\beta_{n}(z)$ represents state functions for a nominal model because they contain only the known terms. In the initial coordinates, their expressions are:

$$
\begin{aligned}
& \alpha_{n}(x)=-\frac{k K_{c} K_{\theta}}{J} \omega\left(\frac{P_{p}}{V_{p}(\theta)}+\frac{P_{n}}{V_{n}(\theta)}\right) \\
& \beta_{n}(x)=\frac{k r T K_{c}}{J}\left(\frac{\varphi_{p}}{V_{p}(\theta)}+\frac{\varphi_{n}}{V_{n}(\theta)}\right)
\end{aligned}
$$

$\Delta_{f}$ and $\delta$ include all the unknown terms, i.e. the leakage mass flow rates, the gravity torque and the dry friction : 
$\Delta_{f}=\frac{k r T K_{c}}{J}\left(\frac{-\dot{m}_{f p}-\dot{m}_{v}}{V_{p}(\theta)}+\frac{\dot{m}_{f n}-\dot{m}_{v}}{V_{n}(\theta)}\right)$

$\delta=\frac{M_{b} g l}{J} \cdot \sin (\theta)-\frac{T_{d r y}}{J}$

$\delta$ represents the difference between the real acceleration and the state variable $z_{3}$ The system dynamic (9) can also be written as:

$\dot{z}=f_{o}(z)+g_{o}(z) A_{u}^{*}+d_{o}$

with: $f_{o}=\left(\begin{array}{c}z_{2} \\ z_{3} \\ \alpha_{n}(z) \\ f_{l}\end{array}\right), g_{o}=\left(\begin{array}{c}0 \\ 0 \\ \beta_{n}(z) \\ g_{l}\end{array}\right)$ and $d_{o}=\left(\begin{array}{c}0 \\ \delta \\ \Delta_{f} \\ 0\end{array}\right)$

$f_{1}$ and $g_{1}$ are the first components of the vectors $f$ and $g$ expressed in the $\mathrm{z}$ coordinates.

The model (13) is equivalent to a nominal dynamic (14) disturbed by $d_{0}$.

$$
\dot{z}=f_{o}(z)+g_{o}(z) A_{u}^{*}
$$

\subsection{Sliding mode controller}

Let:

$* \theta_{d}(t), \omega_{d}(t), \gamma_{d}(t)$ and $\theta_{d}^{(3)}(t)$ be the position, speed, acceleartion and jerk desired trajectories.

$* \tilde{z}=\left(\tilde{z}_{1}, \tilde{z}_{2}, \tilde{z}_{3}\right)=\left(e_{\theta}, e_{\omega}, e_{\gamma}\right)$ be the state error vector containing respectively the position, speed and acceleration errors.

$$
\begin{aligned}
& e_{\theta}=\theta-\theta_{d}(t), e_{\omega}=\omega-\omega_{d}(t), \\
& e_{\gamma}=\frac{K_{c}\left(P_{p}-P_{n}\right)}{J}-\gamma_{d}(t)
\end{aligned}
$$

* $\sigma(\tilde{z})$ is the state surface function.

To synthesize the sliding mode controller two conditions must be held:

- the surface attractivity condition $\sigma \dot{\sigma}<0$ which assure the state errors given above to reach the surface $\sigma=0$ : the sliding mode is then occuring and $\sigma=0$ corresponds to the sliding mode equation.

- when in sliding mode, i.e. for $\sigma=0$, the stability is guranteed under the condition that $\sigma=0 \Rightarrow \tilde{z}_{i}=0 \quad \forall i$.

\section{control synthesis}

The attractivity condition is assured thanks to the following dynamic:

$$
\dot{\sigma}=-W \operatorname{sign}(\sigma)
$$

which, applied to the nominal model (14), leads to the following control:

$$
A_{u}^{*}=-\left(\frac{\partial \sigma}{\partial z} g_{o}\right)^{-1}\left(\frac{\partial \sigma}{\partial z} f_{o}+\frac{\partial \sigma}{\partial t}+W \operatorname{sign}(\sigma)\right)
$$

For the first investigation, the surface is chosen of a second order polynomial (damping factor $=1$ ) :

$$
\sigma(\tilde{z})=\lambda^{2} \tilde{z}_{1}+2 \lambda \tilde{z}_{2}+\tilde{z}_{3}=\lambda^{2} e_{\theta}+2 \lambda e_{\omega}+e_{\gamma}
$$

and the control law is then deduced:

$$
A_{u}^{*}=\frac{-\alpha_{n}(x)-\lambda^{2} e_{\omega}-2 \lambda e_{\gamma}+\theta_{d}^{(3)}-W \operatorname{sign}(\sigma)}{\beta_{n}(x)}(18)
$$

The attractivity condition $\sigma \dot{\sigma}<0$ is maintained on the condition that: $\quad \mathrm{W}>\sup \left(\Delta_{f}+2 \lambda \delta\right)$

The sliding mode equation is then given by:

$$
\left\{\begin{array}{l}
\dot{z}_{1}=\tilde{z}_{2} \\
\dot{\tilde{z}}_{2}=-\lambda^{2}\left(\tilde{z}_{1}-\frac{\delta}{\lambda^{2}}\right)-2 \lambda \tilde{z}_{2}
\end{array}\right.
$$

Assuming that $\delta$ is slowly variable, we easily conclude that the second order system (19) surely leads to a static error $\frac{\delta_{s t a}}{\lambda^{2}}$. $\delta_{\text {sta }}$ corresponds to the static value of $\delta$. $\delta$ is assumed to be slowly variable because we are more interested by the starting and breaking phases. To alleviate this static error, an integral term is added to the surface. Let $\sigma_{I}$ be this new surface which is chosen conforming to Slotine [12].

$$
\begin{gathered}
\sigma_{I}=\left(\lambda+\frac{d}{d t}\right)^{3} \int_{0}^{t} \tilde{z}_{1} d \tau \\
\text { i.e. } \sigma_{I}(z)=\lambda^{3} \int_{0}^{t} e_{\theta} d \tau+3 \lambda^{2} e_{\theta}+3 \lambda e_{\omega}+e_{\gamma}
\end{gathered}
$$

However, the attractivity condition is conditioned by $\mathrm{W}>\sup \left(\Delta_{f}+3 \lambda \delta\right)$.

The corresponding sliding mode controller is synthesized conforming to (16) and is given by:

$$
A_{u_{I}}^{*}=\frac{-\alpha_{n}(x)-\lambda^{3} e_{\theta}-3 \lambda^{2} e_{\omega}-3 \lambda e_{\gamma}+\theta_{d}^{(3)}-W \operatorname{sign}\left(\sigma_{I}\right)}{\beta_{n}(x)}
$$

When in sliding mode the closed loop system dynamic obey to the following equation :

$$
\left\{\begin{array}{l}
\dot{\xi}_{1}=\xi_{2} / \xi_{1}=\int_{0}^{t} e_{\theta} d \tau \\
\dot{\xi}_{2}=\xi_{3} \\
\dot{\xi}_{3}=-\lambda^{3}\left(\xi_{1}-\frac{\delta}{\lambda^{3}}\right)-3 \lambda^{2} \xi_{2}-3 \lambda \xi_{3}
\end{array}\right.
$$

As one can see this dynamic assure the nullity of the static error under the same assumptions that $\delta$ is slowly variable. 
Nota: The equations (19) and (22) correspond to the closed loop system dynamic when in sliding mode in both cases of a classical surface $\sigma$ or an integral surface $\sigma_{\mathrm{I}}$. These equations show that the pressure variable $P_{p}$ is made inobservable by the nonlinear sliding mode control feedback (18) or (21). Its stability was numerically analyzed and shown to be satisfied [3].

\section{Experimental results}

The experimental tests were carried out by measuring the position, the pressures in both the chambers $P_{p}$ and $P_{n}$ and the speed was obtained by numerical derivation on two sampling time.

To reduce chattering in control the discontinuous signum function is replaced by the saturation function which is linear within a boundary layer $\phi$.

$$
\operatorname{sat}(\sigma / \phi)= \begin{cases}\operatorname{sign}(\sigma) & \text { if }|\sigma|>\phi \\ \sigma / \phi & \text { if }|\sigma| \leq \phi\end{cases}
$$

Hence, the sliding mode controllers (18) and (21) need the knowledge of three parameters: the swtching gain $\mathrm{W}$, the surface coeficient $\lambda$ and the boundary layer thickness $\phi$.

$\mathrm{W}$ is chosen relatively to the upper bound of the uncertainty and is adjusted to have an acceptable dynamic.

$\lambda$ fix the closed loop frequency when the sliding mode is occuring and must at least obey to the Shanon theorem. For our case we have chosen it conforming to the following empirical relation [6]:
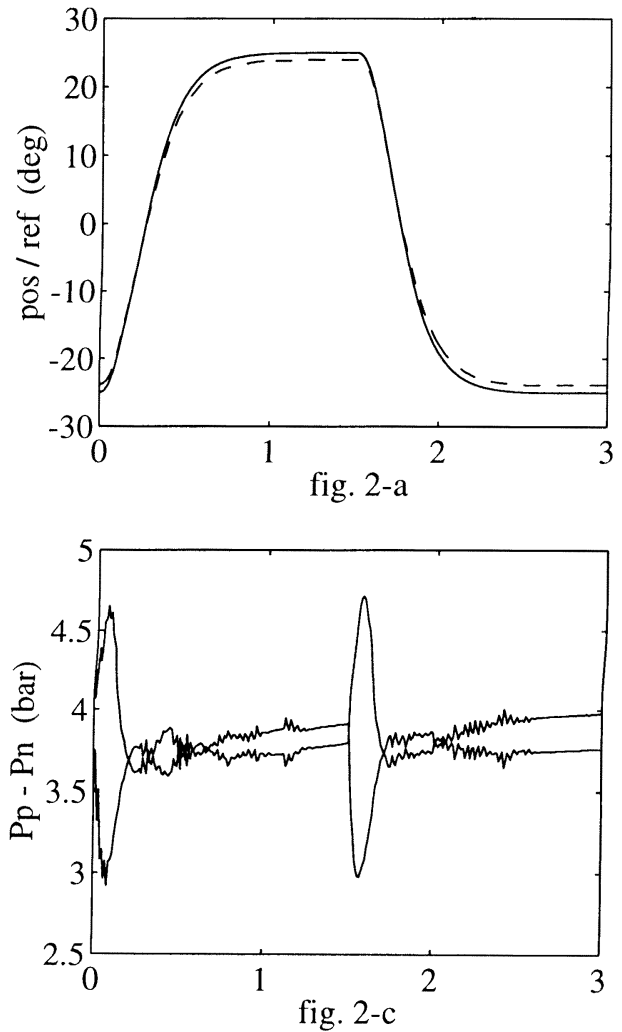

$f_{\text {samp }} \geq \frac{\lambda / 2 \pi}{25} \quad f_{\text {samp }}$ is the sampling frequency

$f_{\text {samp }}=\frac{1}{0,1 m s}=100 \mathrm{hz}, \Rightarrow \lambda \geq 25$

To alleviate chattering due the quantification noise, let $e_{\theta q}, e_{\omega q}$ and $e_{\gamma q}$, be respectively the quantifiocation errors on position, speed and acceleration. We define a quantity $\sigma_{q}$ corresponding to the value of the surface $\sigma$ computed from these quantification errors. The boundary layer $\phi$ is then computed as a sum of $\sigma_{q}$ and a supplementary value $\Delta \phi$ fixed experimentally: $\sigma=\sigma_{q}+\Delta \phi$.

For all the tests and either for the two controllers, $\mathrm{W}$, $\lambda$ and $\phi$ were imposed as: $W=750, \lambda=25$ and $\phi=20$

The desired position is the output of a third order transfer function given by $\frac{1}{(I+0, I s)^{3}}$. The desired speed, acceleration and jerk are deduced.

figure 2-a shows the results obtained by the controller (18) when applying an amplitude of $50^{\circ}$ arround the central position. the dynamic is acceptable and one can see the previous static error (fig. 2-b).

The control is not a lot of chattered (fig. 2-d) and the pressures $P_{p}$ and $P_{n}$ are well stabilized in the static phases (fig. 2-c).
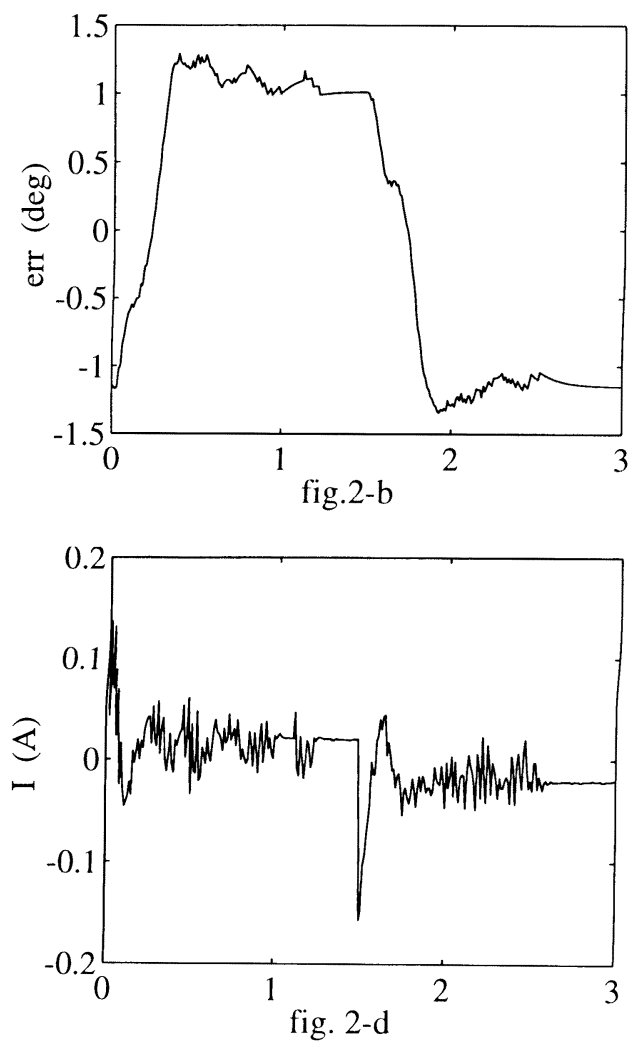
The immediat solution that one can carry out to reduce the static error is to reduce the boundary layer thickness $\phi$. Actually, for $\phi=5$ the static error is well

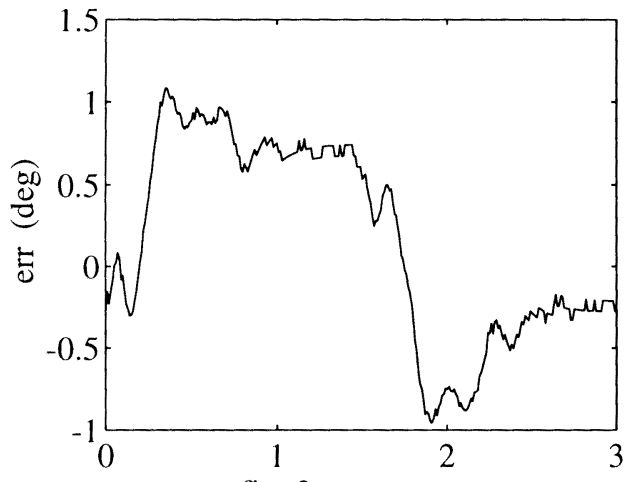

fig. 3-a

More problems appear when tracking trajectories in the extremity. The dynamic is too bad and the static error is important (fig. 4-a,b). This is due to the fact
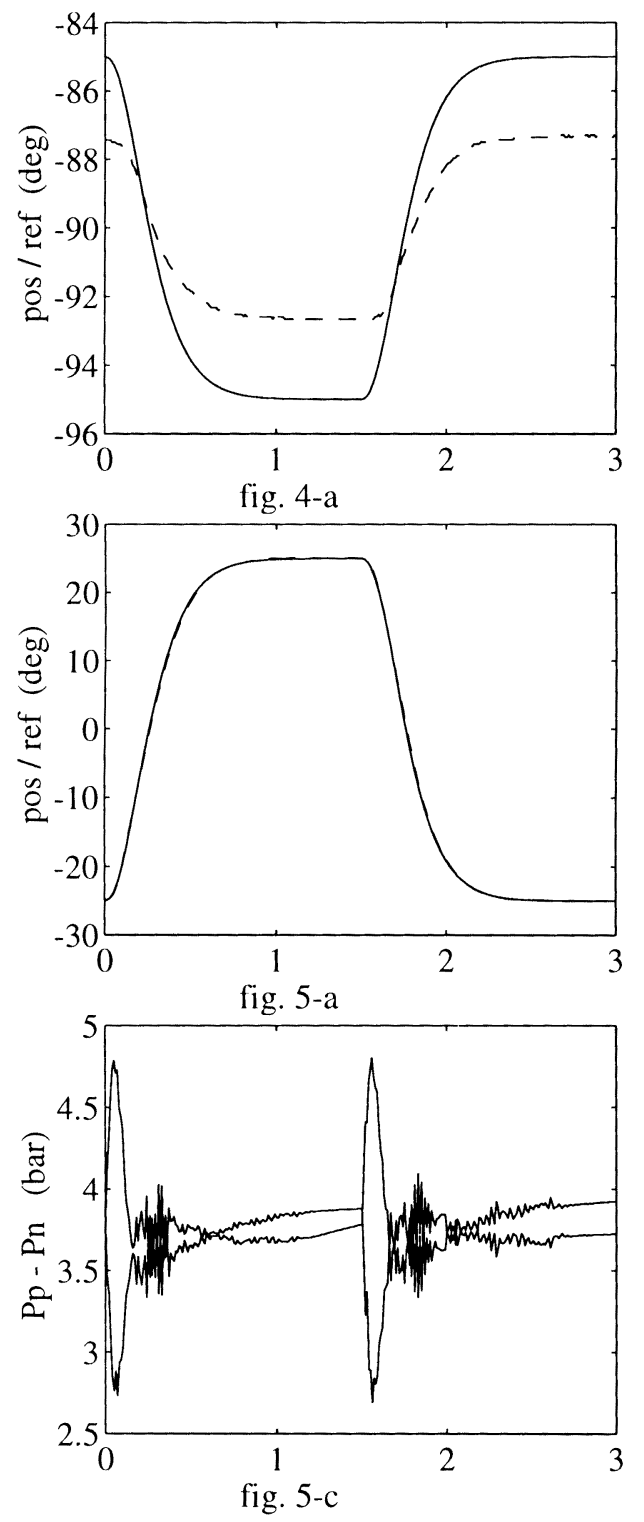

satisfactory (fig. 3-a) at the cost of a lot of chattering in both the output and control (fig. 3-b).

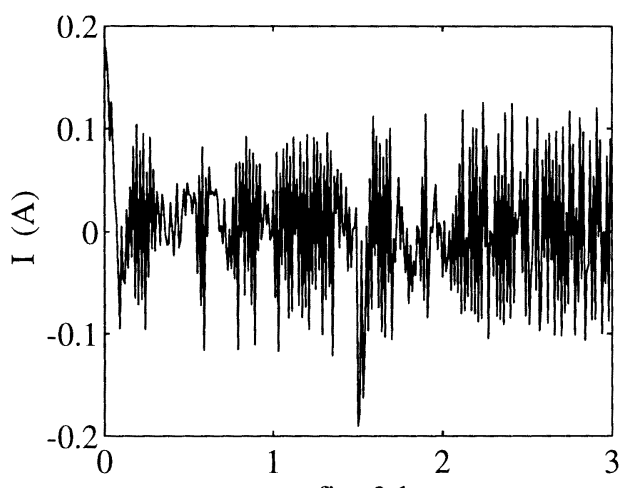

fig. 3-b

that the uncertainties are more important in this case and the parameters must be ajusted once again.
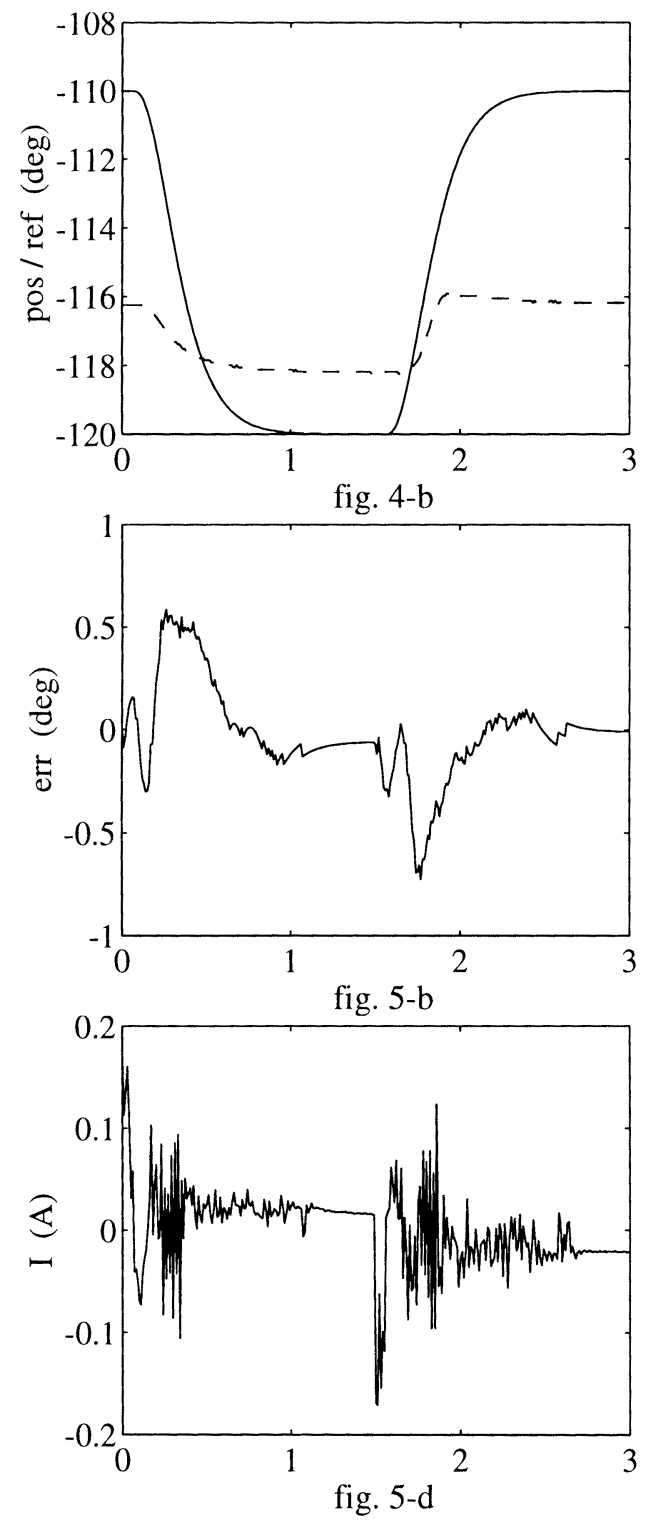
The results obtained with the integral surface are well satisfactory in all cases, i.e. arround the central position (fig. 5-a) and in the extremity (fig. 6-a, 6-b).
As one can see the cost of this improvement is the little increase of chattering in control (fig. 5-d).
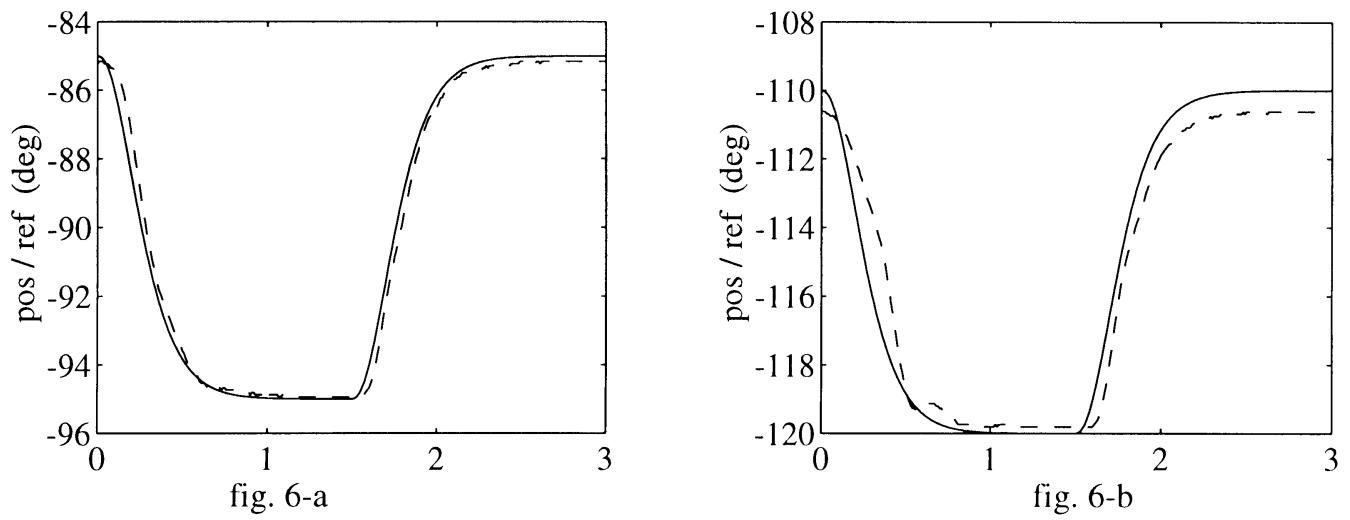

\section{Conclusion}

This paper leads to a synthesis of two nonlinear sliding mode controllers. As expected the sliding controller with an integral term gives more interesting results. The static error is enough decreased relatively to the other controller and the dynamic is satisfactory especially in the extremity of the servodrive. One can easily note the economic impact of this result with respect to the use of the

accelerometer. Actually, the information of the pressure difference was seen to be sufficient to be substituted to the acceleration .

However, in sliding mode control there is no miracle one must always carry out a compromise between chattering and the desired performances and this compromise seemed to be held in our case.

\section{Bibliographie}

[1] Andersen B. W., "The analysis and design of pneumatic systems", John Wiley and Sons, New-York, 1976.

[2] Boudjemaa F. et al., "VSS Approach to A full bridge buck converter used for AC sine voltage generation",

[3] Bouri M., Thomasset D, "On a tracking controller synthesis for nonlinear systems uing a sliding mode approach ", submitted to Int. J. of robust nonlinear control, 1995.

[4] Burrows C. R., "Fluid power servomechanisms ", Van Nostrand Reinhold Company, London, 1972.

[5] Habibi S. R., Richards R. J, "Computed torque and variable structure multivariable conrol of a hydraulic industrial robot", Proc. Instn. Mech. Eng, 205, pp123-140.

[6] Landau I. D. , " Identification et commande des systèmes ", Hermes, 1993.

[7] Lantto et al., "Robust control of an electrohydraulic pump using sliding mode planes", The third Scandinavian International Conference on Fluid Power.

[8] Necsulescu et al., "Low speed motion control of a mechanical system", Dynamics and control, 4, pp307-322, 1993.

[9] Noritsugu T., Wada T., "Adaptive of variable structure control of pneumatically actuated robot", JHPS International Symposium on Fluid Power, Tokyo, March 1989, pp591-598.

[10] Richard E., Scavarda S., " Nonlinear control of pneumatic servodrive ", 2nd Bath fluidpower Workshop, 5975, september 1989.

[11] Scavarda S., "Some theoretical aspects and recent developments in pneumatic positioning systems", Proceedings of the Second JHPS International Symposium on Fluid Power, Tokyo, 6-9 september 1993.

[12] Slotine J. J. E., Li W., "Applied nonlinear control", Prentice Hall, 1991.

[13] Thomasset D., Richard E., Scavarda S., Lin X. F., Sesmat S. ,Bouhal A., " Control of an electropneumatic servodrive: a state affine or a sliding approach ", IFAC World Congress, Sydney (1993). 